\title{
The surface geometry of exotic nuclei
}

\author{
B. V. Carlson*, E. Baldini-Neto*, D. Hirata ${ }^{\dagger}$, S. Péru-Desenfants**, J.-F. \\ Berger** $^{* *}$ C. De Conti ${ }^{\ddagger}$ and L. C. Chamon ${ }^{\S}$ \\ * Departamento de Física do Instituto Tecnológico de Aeronáutica, 12228-901 São José dos \\ Campos, Brazil. \\ ${ }^{\dagger}$ Departament of Physics and Astronomy, The Open University, Milton Keynes, Mk7611 \\ Buckinghamshire, England. \\ ${ }^{* *}$ DPTA/Service de Physique Nucléaire, BP-12-F91680 Bruyères le Châtel, France. \\ ${ }^{\ddagger}$ Unidade Diferenciada de Itapeva, UNESP, São Paulo State University, 18409-010, Itapeva, \\ Brazil. \\ ${ }^{\S}$ Laboratório Pelletron, Instituto de Física da Universidade de São Paulo, CP 66318, 05315-970 \\ São Paulo, Brazil.
}

\begin{abstract}
We analyze the surface geometry of the spherical even-even $\mathrm{Ca}, \mathrm{Ni}, \mathrm{Sn}$ and $\mathrm{Pb}$ nuclei using two approaches: The relativistic Dirac-Hartree-Bogoliubov one with several parameter sets and the non-relativistic Hartree-Fock-Bogoliubov one with the Gogny force. The proton and neutron density distributions are fitted to two-parameter Fermi density distributions to obtain the half-density radii and diffuseness parameters. Those parameters allow us to determine the nature of the neutron skins predicted by the models. The calculations are compared with existing experimental data.
\end{abstract}

Keywords: nuclear structure, relativistic models, surface geometry

PACS: $21.65 .+f, 21.60 .-n, F .21 .60 . J z$

\section{INTRODUCTION}

New experimental facilities have enabled the measurement of masses, radii and deformations in an ever wider region of the nuclear chart. Studies in this 'terra exotica' have revealed new features such as neutron haloes and skins and brought new perspectives to nuclear physics. A more detailed analysis of the variations in surface geometry becomes possible, with the growing number of measurements of the radial distributions of neutrons and protons in nuclei far from stability [1,2].

For heavy neutron-rich nuclei, self-consistent methods, have achieved a level of sophistication and precision which allows analyses of experimental data for a wide range of properties. Those microscopic calculations require a simultaneous description of pairing and continuum effects that can be achieved by mean-field methods like the non-relativistic Hartree-Fock-Bogoliubov approach (HFB) with a Skyrme or Gogny interaction [3, 4], or relativistic Dirac-Hartree-Bogoliubov (DHB) approach [5](and references there in).

In this work, we analyze the surface geometry in of the even-even isotopic chains of the spherical $\mathrm{Ca}, \mathrm{Ni}, \mathrm{Sn}$ and $\mathrm{Pb}$ nuclei, using the relativistic Dirac-Hartree-Bogoliubov (DHB) with several parametrizations and the non-relativistic HFB+Gogny (HFB) approximations. The proton and neutron densities obtained from the calculations are fitted by two-parameter Fermi density distributions to extract the half-densities, and rms radii as well as their diffuseness parameters. The results are compared with each other and

CP884, VI Latin American Symposium on Nuclear Physics and Applications,

edited by O. Civitarese, C. Dorso, G. García Bermúdez, A. J. Kreiner, A. J. Pacheco, and N. N. Scoccola (C) 2007 American Institute of Physics 978-0-7354-0388-8/07/\$23.00 
also with the experimental data [6]. The calculated differences between neutron and proton rms radii, $\Delta r_{n p}$, as a function of the asymmetry parameter $\delta=\frac{N-Z}{A}$ are discussed and compared with those obtained from the systematics given in Ref. [2].

\section{THEORETICAL FORMALISM}

\section{The Dirac-Hartree-Bogoliubov approximation (DHB)}

Relativistic many-body theories have been applied to nuclei and nuclear matter with remarkable success [7] (and references therein). The DHB approximation is obtained by taking into account the average effect of the interaction of a nucleon with the other nucleons through an effective single-particle Lagrangian, given in terms of a self-energy $\Sigma_{t}(\vec{x}, \vec{y})$, which describes the average interaction of a nucleon with the surrounding matter, and a pairing field $\Delta_{t}(\vec{x}, \vec{y})$ (and its conjugate $\bar{\Delta}$ ) which describes the creation (annihilation) of a pair during the propagation.

The DHB approximation used here was studied previously [5] and found to give a good description of the binding energies, rms radii and deformations in a large range of nuclei. It contains a free and interacting Lagrangians with nucleons, $\sigma, \omega, \rho$ mesons and Coulomb fields as well as a non-linear potential or density dependent coupling constants and rearrangement terms. The DHB equations are solved self-consistently together with the meson fields that are solutions of Klein-Gordon equations. Both fields and wavefunctions are expanded in a complete set of eigenfunctions of an axially-deformed harmonic oscillator potential, with several parameter sets, namely, the NL3 interaction [8], the density-dependent DDME1 force [9], the non-linear PK1 and density-dependent PKDD forces [10] and a zero-range Hartree-Fock interaction with finite-range corrections, DHFB [11], using 16 major fermion shells and 32 major boson shells.

\section{Hartree-Fock-Bogoliubov with the Gogny interaction (HFB)}

The Gogny interaction [12] was originally proposed to describe the mean-field and pairing properties on the same footing within the HFB theory. One of its advantages in relation to a zero-range pairing interaction is the elimination of divergences that can occur when using the latter. This interaction has 13 adjustable parameters well known from the literature $[12,13]$. The parameters used are those of the D1S version of the force, given in the Appendix of Ref. [3]. The HFB equations are solved by expanding the quasi-particle states on finite sets of an harmonic oscillator basis containing 13 shells in $\mathrm{O}$ and $\mathrm{Ca}, 15$ shells in $\mathrm{Ni}$ and 17 shells in $\mathrm{Pb}$.

\section{RESULTS}

Results of the calculations are used as input for the two-Fermi density distributions least-square fitting. The diffuseness parameter and half-density radii are then extracted. 

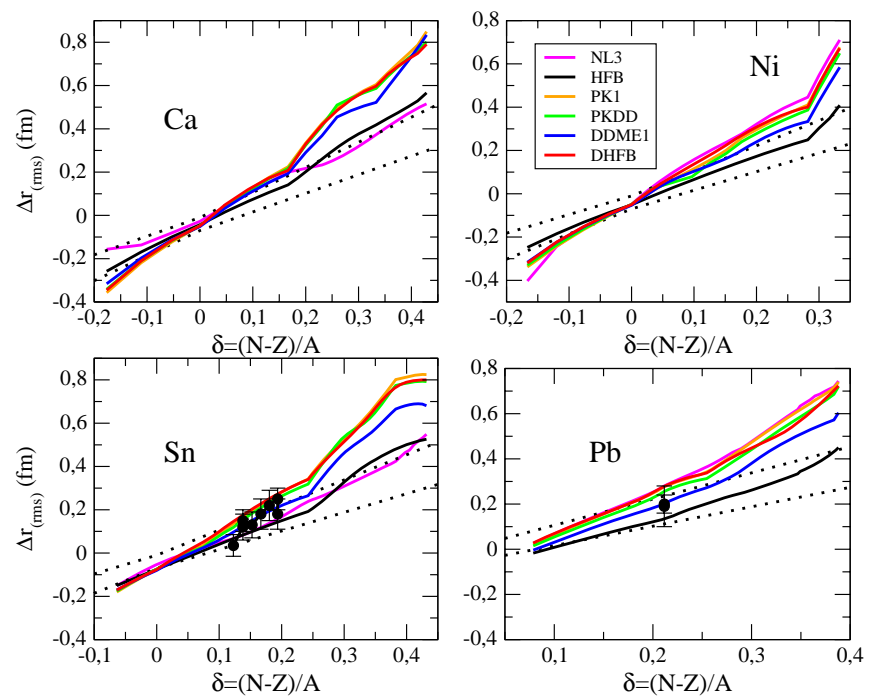

FIGURE 1. The several DHB calculations and the HFB one together with the systematics (delimited by dotted-lines) for differences between neutron and proton rms radii as a function of the asymmetry parameter. The experimental data for $\mathrm{Sn}$ and $\mathrm{Pb}$ are also shown.

The rms radii are determined directly from the neutron and proton distributions. The dependence of the difference between neutron and proton rms radii of the DHB and HFB calculations on the asymmetry parameter, $\delta=(N-Z) / A$ is shown in Fig. 1. Our results are compared with the ones obtained from systematics, $\Delta r_{s y s}$ [2]. The HFB calculations lie, for the most part, between the maximum and minimum values of $\Delta r_{s y s}$, extending beyond the upper limit of the systematics only for extremely large values of the asymmetry parameter. The DHB calculations in most cases deviate substantially from the systematics at high neutron excess.

The diffuseness parameter $(a)$ as a function of mass $(A)$ for even-even $\mathrm{Ca}, \mathrm{Ni}, \mathrm{Sn}$ and $\mathrm{Pb}$ isotopes is shown in Fig. 2. The diffuseness extracted from the fitting of DHB and HFB protons density distributions are connected by dashed lines, while the full lines represent the neutron diffuseness parameters. The experimental data shown in the figure are obtained from the two-parameter Fermi $(2 \mathrm{pF})$, three-parameter Fermi $(3 \mathrm{pF})$ and threeparameter Gaussian (3pG) fits to elastic electron scattering data, as compiled in Ref. [14]. The density distributions given there were fitted with a two-parameter Fermi distribution convoluted with the proton charge distribution to obtain the half-density radii and diffuseness parameters shown as data points in the figures. The DHB calculations tend to provide slightly smaller neutron diffuseness parameters than the ones obtained from the HFB calculations for low neutron excess. The proton diffuseness parameters in the relativistic calculations are smaller then those calculated relativistically. One notes that, as a closed shell is approached the nucleons become more bound on the average and the diffuseness parameter decreases (excepting the case of $\mathrm{Pb}$ ), increasing again as a new shell opens. 

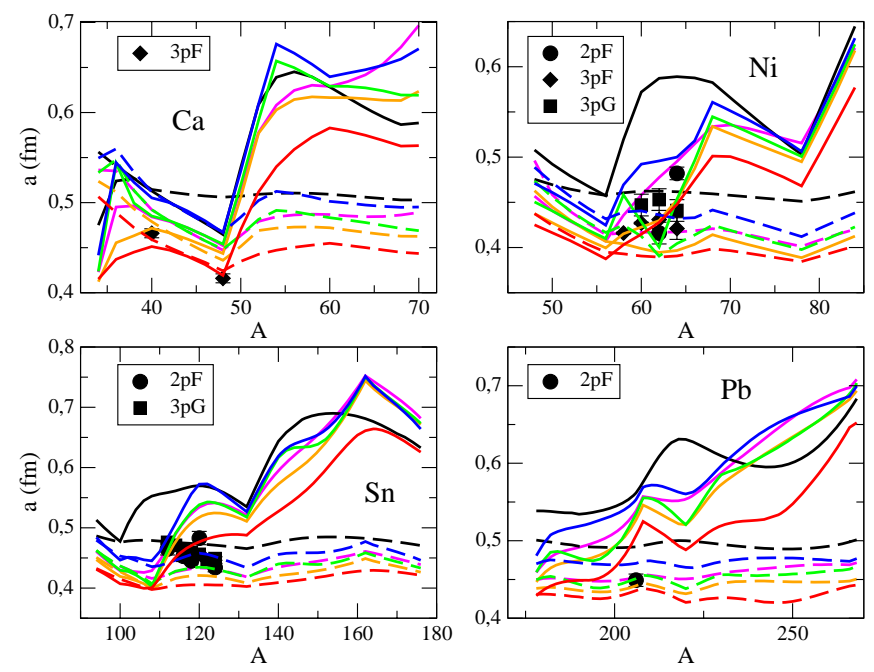

FIGURE 2. Calculations for difuseness parameters for neutrons (full lines) and protons (dashed lines) for HFB calculations and several parametrizations of the DHB approach, compared with the experimental data when available. The legends are the same as the previous figure.

Similar features are found when the behavior of the half-density radii is analyzed. Figure 3 presents the half-density radius $(c)$ as a function of mass $(A)$ for the eveneven $\mathrm{Ca}, \mathrm{Ni}, \mathrm{Sn}$ and $\mathrm{Pb}$ isotopes. In each isotopic chain we can see that the half-density radii for both protons and neutrons increase smoothly with the increase in mass, in both calculations.

The shell closure effects are more pronounced in the diffuseness parameters extracted from the DHB calculations while these effects are more prominent in the half-density radii extracted from the HFB ones, as seen in Fig. 2 and Fig. 3.

\section{CONCLUSIONS}

A study of the surface geometry (diffuseness and half-density radii) by a least-square fitting of neutron and proton density distributions of spherical nuclei has been performed. The density distributions of the spherical neutron-rich even-even mass $\mathrm{Ca}, \mathrm{Ni}$, $\mathrm{Sn}$ and $\mathrm{Pb}$ isotopic chains have been adjusted to two-parameter Fermi density distributions. Both neutron and proton half-density radii increase fairly smoothly towards the neutron drip-line in all calculations. A substantial increase in the diffuseness parameter in isotopes far from the stability line is seen in all calculations. In general, the diffuseness parameters decrease at the last sub-shell before the magic number is reached. The only exception occurred for $\mathrm{Pb}$ isotopes.

The DHB approach greatly overestimates the neutron and proton rms radius difference at large neutron excess when compared to the systematics of Ref. [2]. The HFB approach shows a better agreement with the empirical systematics for the $\Delta r_{n p}$ differences. 

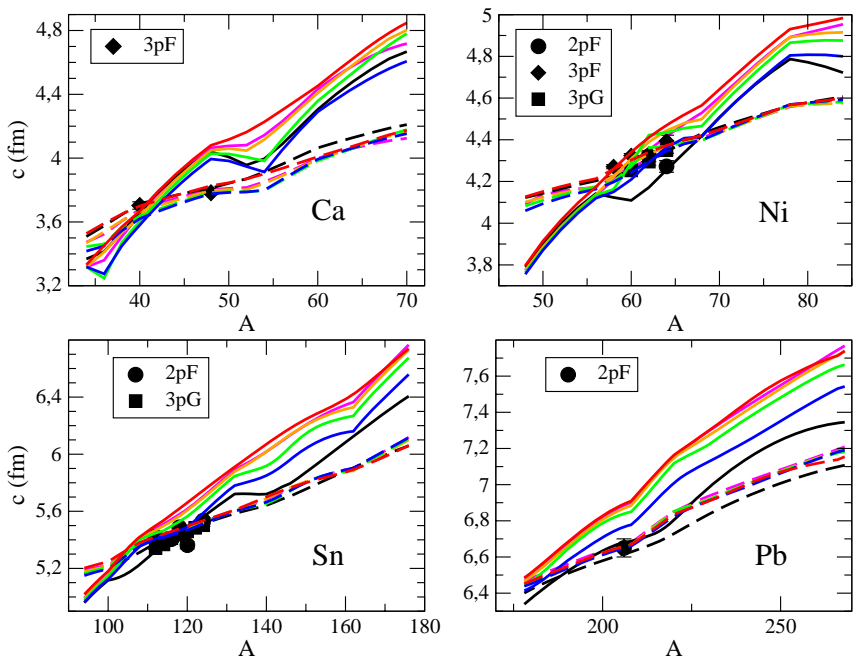

FIGURE 3. Calculations for half-density radii for neutrons (full line) and protons (dashed lines) for HFB calculations and several parametrizations of the DHB approach, compared with the experimental data when available. The legends are the same as in Fig. 1

\section{ACKNOWLEDGMENTS}

EBN and DH would like to thank FAPESP, Fundação de Amparo à Pesquisa do Estado de São Paulo, for finnancial support. BVC and LCC acknowledge partial support from FAPESP and CNPq.

\section{REFERENCES}

1. T. Suzuki et al. Phys. Rev. Lett. 75, 3241 (1995).

2. A. Trzcińska, J. Jastrezę, P. Lubiński, F. J. Hartmann, R. Schmidt, T. von Egidy and B. Klos, Phys. Rev. Lett. 87, 082501 (2001).

3. J.-F. Berger, M. Girod and D. Gogny, Comput. Phys. Comm. 63, 365 (1991).

4. J. Dobaczewski, W. Nazarewicz, T. R. Werner, J.-F. Berger, C. R. Chinn and J. Dechargé, Phys. Rev. C 53, 2809 (1996).

5. B. V. Carlson and D. Hirata, Phys. Rev. C 62054310 (2000).

6. G. Audi, O. Bersillon, J. Blachot and A. H. Wapstra, Nucl. Phys. A 729, 3-128 (2003).

7. B. D. Serot and J. D. Walecka, Adv. Nucl. Phys. 16, 1 (1986).

8. G. A. Lalazissis, J. König and P. Ring, Phys. Rev. C 55, 540 (1997).

9. T. Niksic, D. Vretenar, P. Finelli and P. Ring, Phys. Rev. C 66, 024306, (2002).

10. W. Long, J. Meng, N. Van Giai and S. -G. Zhou, Phys. Rev. C 69, 034319 (2004).

11. B. V. Carlson, D. Hirata, unpublished.

12. D. Gogny, Proceedings of the International Conference on Nuclear Physics, edited by J. de Boer and H. J. Mang, North-Holland, Amsterdam, 1973, p. 48.

13. J. Dechargé and D. Gogny, Phys. Rev. C 21, 1568 (1980).

14. H. de Vries, C. W. de Jager and C. de Vries, Atomic data and nuclear data tables 36, 495 (1987). 
Copyright of AIP Conference Proceedings is the property of American Institute of Physics and its content may not be copied or emailed to multiple sites or posted to a listserv without the copyright holder's express written permission. However, users may print, download, or email articles for individual use. 